\title{
Comparison of two methods for correcting baseline offset error in phase-contrast MR imaging
}

\author{
Yang Lin ${ }^{12^{*}}$, Ke Jiang ${ }^{1}$, Yiu-Cho Chung ${ }^{1}$ \\ From 19th Annual SCMR Scientific Sessions \\ Los Angeles, CA, USA. 27-30 January 2016
}

\section{Background}

Phase-contrast MRI (PC-MRI) can be used to assess valvular heart diseases. It can also measure pulmonarysystemic flow ratio (Qp/Qs) and help identify intracardiac shunts. However, phase offsets in PC-MRI caused by main field inhomogeneity and eddy-current introduce baseline offset errors in flow quantification and hence $\mathrm{Qp} / \mathrm{Qs}$. This error can be corrected by surface-fitting [1] or using a separate phantom acquisition [2]. A recent study found that the phantom acquisition method did not help reduce error of Qp/Qs [3]. This study compared the effectiveness of the two baseline correction methods in reducing phase errors when measuring Qp/Qs in healthy volunteers using PC-MRI.

\section{Methods}

Ten healthy volunteers were recruited for this IRB approved study. Each volunteer gave informed consent. The study was performed on a 3.0 T MRI clinical scanner (Trio Tim, Siemens, Germany). In each subject, localizers were used to find the aortic and pulmonary outflow tract. Velocity encoded, retrogated gradient echo cine was used to measure blood flow perpendicular to the two outflow tracts. Imaging parameters were: TR/ $\mathrm{TE}=4.4$, flip angle $=20^{\circ}$, slice thickness $=5.5 \mathrm{~mm}$, matrix size $=192 \times 144$, bandwidth $=704 \mathrm{~Hz} /$ pixel,

$\mathrm{VENC}=170 \mathrm{~cm} / \mathrm{s}, 5$ lines per heartbeat, 20 reconstructed phases. After flow measurements, a water phantom was put inside the scanner. It was scanned with identical flow imaging protocols after waiting for five minutes [4]. Baseline correction using phantom was performed following [3]. Baseline correction by the surfacefitting method was performed using Qflow (Medis, Netherland). Qp/Qs was calculated for each volunteer.

\section{Results}

Table 1 lists the mean and standard deviation of Qp/Qs ratios before and after correction using the two methods. The surface-fitting method brought the Qp/Qs ratio closer to 1 than the phantom method. Figure 1 shows how the two correction methods changed the $\mathrm{Qp} / \mathrm{Qs}$ ratio in individual cases. Qp/Qs ratios were lower $(\mathrm{p}<0.05)$ after being corrected by the surfacefitting method. Reduction of Qp/Qs ratios using the phantom correction method was not statistically significant $(\mathrm{p}=0.1)$.

\section{Conclusions}

The surface-fitting method reduced the Qp/Qs ratios in all cases while the phantom correction method increased the ratio in one case. As the phantom correction method needs additional scanning time, the surface-fitting

Table 1 Summary of aortic flow, pulmonic flow and Qp/Qs before and after baseline correction

\begin{tabular}{cccc}
\hline & Aortic flow $(\mathrm{mL})$ & Pulmonic flow $(\mathrm{mL})$ & Qp/Qs \\
\hline Before correction & $86.0 \pm 15.1$ & $99.6 \pm 19.6$ & $1.16 \pm 0.06$ \\
After phantom correction & $86.5 \pm 14.7$ & $98.1 \pm 19.1$ & $1.13 \pm 0.06$ \\
After surface-fitting correction & $87.1 \pm 15.4$ & $96.2 \pm 18.6$ & $1.10 \pm 0.05$ \\
\hline
\end{tabular}

'Shenzhen Institutes of Advanced Technology, Chinese Academy of

Sciences, Shenzhen, China

Full list of author information is available at the end of the article 


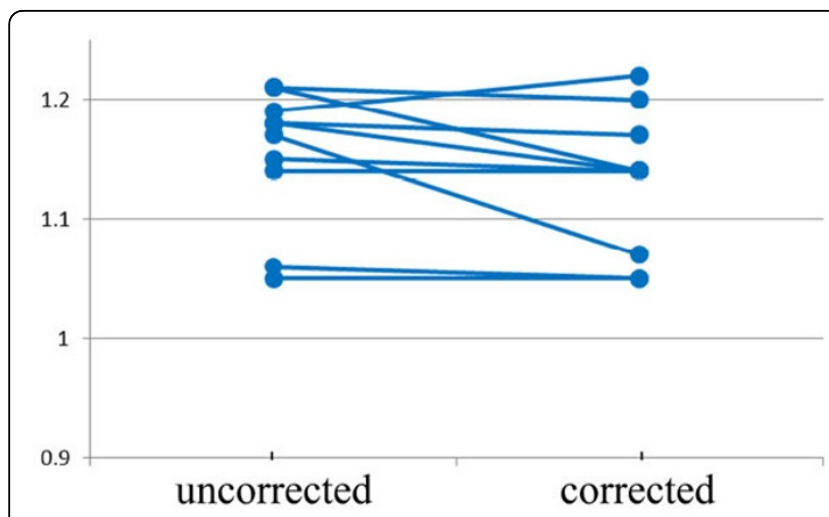

(a) Phantom correction

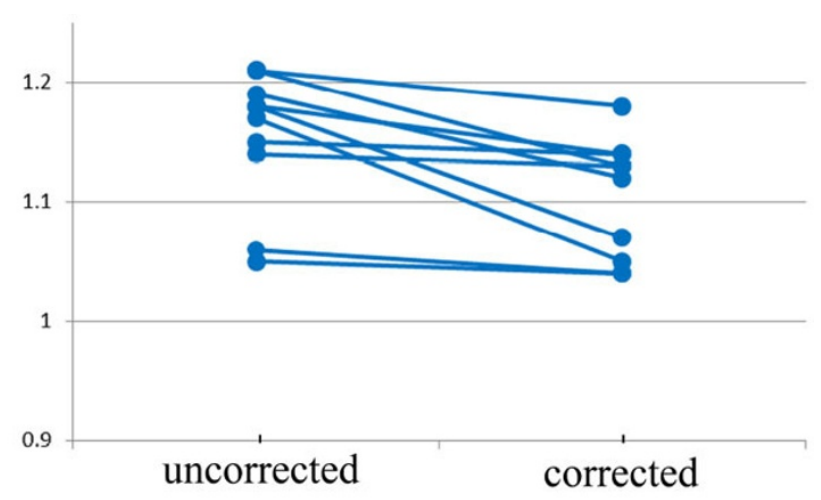

(b) Surface-fitting correction

Figure $1 \mathrm{Qp} / \mathrm{Qs}$ ratio of the healthy volunteers before and after baseline correction. (a) Correction using phantom; (b) correction using surface-fitting.

approach would be preferred for baseline offset correction in PC-MRI.

\section{Authors' details}

'Shenzhen Institutes of Advanced Technology, Chinese Academy of

Sciences, Shenzhen, China. ${ }^{2}$ University of Science and Technology of China,

Hefei, China.

Published: 27 January 2016

\section{References}

1. Lankhaar JW, et al: JMRI 2005, 22(1):73-79.

2. Chernobelsky A, et al: JCMR 2007, 9(4):681-685.

3. Meierhofer C, et al: Clinical Imaging 2015, 39(3):427-431.

4. Holland BJ, et al: JCMR 2010, 12(11)

doi:10.1186/1532-429X-18-S1-P334

Cite this article as: Lin et al:: Comparison of two methods for correcting

baseline offset error in phase-contrast MR imaging. Journal of

Cardiovascular Magnetic Resonance 2016 18(Suppl 1):P334.

Submit your next manuscript to BioMed Central and take full advantage of:

- Convenient online submission

- Thorough peer review

- No space constraints or color figure charges

- Immediate publication on acceptance

- Inclusion in PubMed, CAS, Scopus and Google Scholar

- Research which is freely available for redistribution

Submit your manuscript at www.biomedcentral.com/submit 